\section{$\underset{\substack{\text { hommes } \\ \text { \& migrations }}}{ }$}

\section{Hommes \& migrations}

Revue française de référence sur les dynamiques

migratoires

$1311 \mid 2015$

Femmes et migrations

\title{
Femmes camerounaises en migration
}

De l'invisibilité institutionnelle à un dynamisme migratoire accéléré

\section{Pierre Kamdem}

\section{(2) OpenEdition \\ 1 Journals}

\section{Édition électronique}

URL : http://journals.openedition.org/hommesmigrations/3300

DOI : 10.4000/hommesmigrations.3300

ISSN : 2262-3353

Éditeur

Musée national de l'histoire de l'immigration

\section{Édition imprimée}

Date de publication : 1 juillet 2015

Pagination : 115-121

ISBN : 978-2-919040-32-2

ISSN : 1142-852X

Référence électronique

Pierre Kamdem, «Femmes camerounaises en migration », Hommes \& migrations [En ligne],

1311 | 2015, mis en ligne le 01 juillet 2018, consulté le 19 avril 2019. URL : http://

journals.openedition.org/hommesmigrations/3300 ; DOI : 10.4000/hommesmigrations.3300 


\title{
FEMMES CAMEROUNAISES EN MIGRATION DE L'INVISIBILITÉ INSTITUTIONNELLE À UN DYNAMISME MIGRATOIRE ACCÉLÉRÉ
}

par PIERRE KAMDEM, enseignant-chercheur, HDR en géographie, université de Dschang (Cameroun).

\author{
La féminisation des flux migratoires au départ du Cameroun \\ s'est accélérée depuis le début des années 1990. Dans leur pays \\ d'accueil, les migrantes camerounaises déploient différentes \\ stratégies de visibilité, dont les actions se manifestent \\ aux niveaux culturel, économique et politique. En Île-de-France, \\ par exemple, elles sont à la fois des passeuses reconnues \\ de leur culture et des actrices économiques de premier plan. \\ Éléments clés de la structuration des espaces associatifs \\ en migration, ces femmes luttent contre les pesanteurs sociales \\ et les discriminations de genre.
}

Issu d'une construction territoriale assez particulière, le Cameroun présente un contexte migratoire tout aussi spécifique ${ }^{1}$. L’effet de diverses hégémonies impérialistes et coloniales a conduit à l'adoption de modes migratoires assez variés. Bien que son solde migratoire apparaisse officiellement positif, ce territoire s'érige en un pourvoyeur de plus en plus important de migrantes internationales. Le regroupement familial et l'appel du marché du travail (les emplois domestiques, les emplois liés aux soins et à la prise en charge de personnes et la prostitution) constituent les principaux facteurs de mobilité des Camerounaises. Les caractères stratégique et collectif de la migration affectent, par ailleurs, la prise de décision migratoire. Il s'agit d'interroger les différentes conditions qui sous- tendent autant la mise en migration et l'accueil, que le retour des femmes camerounaises, afin de mieux comprendre les stratégies et les postures de visibilité dont elles font preuve dans les divers espaces migratoires pratiqués.

\section{Des dispositifs de mise en migration peu favorables aux femmes}

Les prémices de la mise en migration se dessinent au Cameroun durant la période coloniale. Les nombreuses églises, principalement protestantes soutenues par la Baptist Missionary Society², qui s'installent en particulier dans la partie méridionale 
du pays tout au long de la deuxième moitié du XIX siècle, engagent des stratégies de construction de clergé particulièrement focalisées sur les pasteurs masculins. Le système colonial de la première moitié du XXe siècle s'intéresse davantage aux hommes au titre de leur force de travail et de la constitution d'un vivier de main-d'œuvre ${ }^{3}$, que ce soit dans le cadre des migrations internes au service des grands travaux et chantiers (chemin de fer Congo-Océan et plantations coloniales) ou des migrations internationales pour la formation d'auxiliaires de l'administration coloniale. Le caractère fortement disputé de la situation stratégique

L'imposition du thème de la promotion féminine dans les agendas internationaux dès la conférence de Mexico en 1975 et le lancement de la décennie de la femme

(1975-1985) semblent avoir insufflé au Cameroun un élan féministe. du Cameroun par les principales puissances coloniales tout au long de la période critique du XX siècle confine la gestion des mouvements de population dans un cadre prioritairement sécuritaire ${ }^{4}$. Les mouvements de rébellion face à l'ordre colonial imposent un encadrement réglementaire particulièrement rigide des déplacements des personnes, visant principalement les hommes. Il est clair que l'insécurité ambiante de la période ne fut pas de nature à promouvoir les mobilités féminines. Par ailleurs, les femmes n'étaient guère mieux considérées en métropole. Elles ne sont devenues officiellement électrices et éligibles en France, dans les mêmes conditions que les hommes, qu'en 1944. Bien que le Cameroun ait été l'un des premiers pays africains à le faire dès $1946^{5}$, les conditions réelles d'application ne connurent pas de véritable incidence, même au-delà de l'indépendance survenue en 1960 .

Le vent de liberté qu'apporte l'indépendance camerounaise s'avère vicié par la poursuite des actions des mouvements indépendantistes initiées depuis la fin de la Deuxième Guerre mondiale, inscrivant littéralement le Cameroun dans le théâtre de l'affron- tement des deux blocs. Un pouvoir en place acquis à la cause occidentale, combattant une rébellion marxiste, prolonge les procédures d'encadrement de la population par des politiques de gestion des flux migratoires axées sur les limitations de sorties du territoire, avec l'instauration du passeport fédéral en 1962. Malgré la suppression de certaines institutions coutumières telles que le lévirat, le sororat et la répudiation, par l'ordonnance 81/02 du 29 juin 1981 portant organisation de l'état civil au Cameroun, ces contraintes persistent pour les femmes à travers, par exemple, l'instauration de l'autorisation maritale de sortie du pays. L'emprise masculine déjà assez pesante à travers, entre autres, le nonversement de l'allocation logement aux femmes actives mariées, s'en trouve renforcée. L'imposition du thème de la promotion féminine dans les agendas internationaux dès la conférence de Mexico en 1975 et le lancement de la décennie de la femme (1975-1985) semblent avoir insuffé au Cameroun un élan féministe. L'une de ses répercussions va être la féminisation des flux migratoires camerounais, observée dès la fin des années 1980. Cette tendance est renforcée non seulement par la crise d'un pouvoir camerounais exclusivement masculin ${ }^{6}$ au tout début des années 1990, mais aussi par la réaction des femmes. Les rapports sociaux de sexe en vigueur dans le pays, conférant traditionnellement aux hommes, selon Pierre Bourdieu "le monopole (...) de l'humain universel", sont remis en cause.

\section{Bouleversements de la société camerounaise et évolution des profils migratoires}

La libéralisation politique et la transition démocratique se concrétisent pour les Camerounaises par l'abrogation de l'ensemble des dispositifs limitatifs dans le contexte de la promulgation des lois de

3. Martin Zachary Njeuma, Histoire du Cameroun. XIXe-début XXe siècle, Paris, L'Harmattan, 2000. 4. Pascal Dejoli Mbogning, "La politique migratoire du Cameroun : la rigidité normative à l'épreuve des flux humains", in Luc Sindjoun (dir.), État, individus et réseaux dans les migrations africaines, Paris, Karthala, 2002, pp. 19-66. 5. Programme des Nations unies pour le développement (PNUD), "Rapport mondial sur le développement humain", 2002 [en ligne]. 6. Aussi qualifiée de crise de la "gouvernementalité phallocratique" par Luc Sindjoun, La Biographie sociale du sexe. Genre, société et politique au Cameroun, Paris, Codesria/Karthala, 2000. 7. Pierre Bourdieu, "La domination masculine", in Actes de la recherche en sciences sociales, n 84, 1990, pp. 2-31. 
démocratisation en 1990, suivie de la ratification par le Cameroun, le 23 août 1994, de la Convention sur l'élimination de toutes les formes de discriminations à l'égard des femmes (Cedef), aussi appelée "charte internationale des droits de la femme". Autant de leviers qui stimulent l'irruption des femmes dans les flux migratoires au départ du Cameroun. Dans le même temps, les plans d'ajustements structurels portent un sérieux coup aux structures familiales ${ }^{8}$ en laminant le pouvoir d'achat masculin et, par ricochet, leur position sociale traditionnellement dominante. Apparaissent alors de nombreux facteurs de changement tels que l'école, les médias, les mouvements associatifs et autres réseaux de solidarité. Ils génèrent de nouvelles valeurs à l'instar de la monoparentalité, des femmes chefs de ménage, de l'instabilité matrimoniale et des familles recomposées, qui constituent autant de sources de diversification des stratégies de sortie de crise ancrées dans la mobilité.

Les migrantes partent principalement de Yaoundé et de Douala, les deux grandes métropoles du pays. Elles sont directement ou indirectement issues des intenses flux migratoires internes nourris par l'exode rural qui affecte les régions administratives ${ }^{9}$ de la partie méridionale du pays. Les trois principales régions émettrices sont, par ordre d'importance, l'Ouest, le Centre et le Littoral. Les mouvements de ces femmes sont, par ailleurs, fortement impactés par certains paramètres tels que les réseaux migratoires préalablement installés en zone de destination, pour celles qui sont prises en charge par des membres de leur famille ou des amis déjà installés à létranger. Les plus mobiles se recrutent dans la tranche 25-29 ans. En général, les femmes âgées qui partent du Cameroun ont accusé soit des problèmes de santé, soit des problèmes dans leur ménage. Les plus jeunes, quant à elles, se déplacent beaucoup plus pour des raisons d'études, de formation ou de travail.

Les Camerounaises se dirigent en priorité vers les pays qui ont un lien colonial avec le Cameroun, à savoir l'Angleterre pour les anglophones et la France pour les francophones, faisant ainsi de l'Europe leur principale destination. Toutefois, le

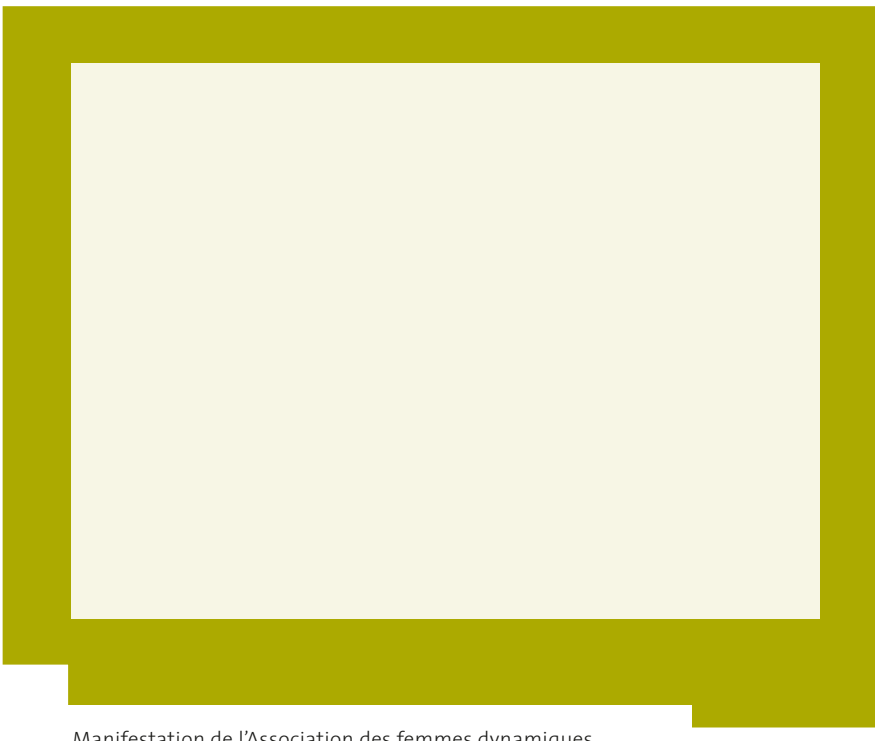

Manifestation de l'Association des femmes dynamiques, branche du Rassemblement démocratique du peuple camerounais (RDPC). (c) PIERRE KAMDEM.

durcissement des conditions d'obtention des visas d'entrée dans l'Union européenne suscite la prospection d'autres zones d'accueil telles que l'Amérique et l'Asie, tout en relançant les destinations intra-africaines telles le Gabon, ou encore plus récemment la Guinée équatoriale.

L'Europe et l'Amérique sont surtout des destinations pour études. L'augmentation du niveau d'instruction va de pair avec celle du capital social, économique et infrastructurel. Elle favorise l'émigration internationale des Camerounaises comme constaté dansles flux migratoires vers l'Allemagne ${ }^{10}$. 
C'est dans cette ambiance générale que se dessine la visibilité des Camerounaises en migration, dont il s'agit d'interroger les stratégies en Île-de-France, selon trois dimensions : socioculturelle, socio-économique et sociopolitique.

\section{Stratégies de visibilité des Camerounaises en migration : le poids de la culture}

La visibilité des Camerounaises en migration peut s'analyser sous deux aspects, l'un direct et l'autre indirect. Moins prégnante, la visibilité directe consiste dans la mise en scène immédiate des migrantes, à travers des activités genrées telles que les formes entrepreneuriales fortement féminisées et parfois ethniques comme la restauration exotique, les salons de coiffure et d'esthétique, les services à la personne, marquant spatialement et socialement ce courant migratoire. La visibilité indirecte rend compte des situations spatiales et sociales subissant de fortes influences féminines, quand elles n'en dépendent pas tout simplement. La première dimension, socioculturelle, opère autant sur la visibilité directe des migrantes camerounaises que sur leur visibilité indirecte. Elle s'appuie sur un ensemble d'éléments de la culture du groupe d'origine. L'un de ses piliers reste la cuisine, fortement chevillée à l'identité féminine en pays d'origine ${ }^{11}$. En effet, la cuisine et le fait alimentaire constituent des marqueurs culturels majeurs qui participent très activement à la fabrique des identités. En pays d'origine, c'est l'apanage de la gent féminine à laquelle incombe alors le devoir de transposer et de transmettre en migration. Ainsi, les fêtes et autres rituels à caractère traditionnel

\section{/// Figure 1: Origines ethniques des partenaires de Camerounais en île-de-France.}

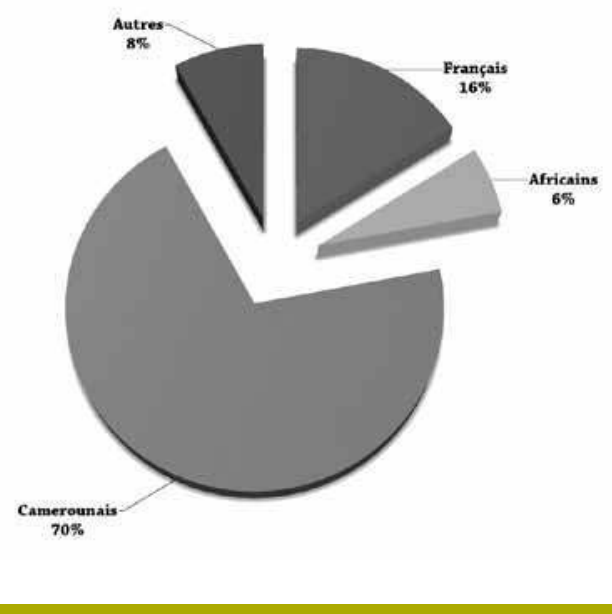

Source : Pierre Kamdem, Camerounais en île-de-France. Dynamiques migratoires et stratégies d'intégration socio-spatiale différenciées, Paris, L'Harmattan, 2007. (Sur la base d'un échantillon de 229 personnes issu du fichier électronique des abonnés France Télécom 2002-2003.

À cette époque, cette entreprise détenait encore le monopole des lignes téléphoniques fixes.)

en migration s'agrémentent de mets locaux dont la confection est assurée par les migrantes. Ces célébrations concourent non seulement à la dynamisation du mouvement associatif camerounais, dont ces migrantes constituent des actrices majeures ${ }^{12}$, mais aussi au renforcement de l'espace associatif par ce devoir de transmission, tout comme leur capacité à générer un marché matrimonial endogamique $^{13}$ participant à la fabrique de la grégarité en migration. Un autre point de consolidation culturelle de la communauté camerounaise se situe dans les danses traditionnelles dont les femmes 
sont censées détenir les codes. Elles sont aussi censées être dépositaires de la validation de certaines procédures inhérentes à l'acquisition de la notabilité traditionnelle, qui demeure célébrée en migration. Elles sont ainsi les garantes de la régularité et de l'authenticité des pratiques traditionnelles en migration.

Les migrantes camerounaises jouent un rôle déterminant dans la dynamique associative. Cette dernière est convoquée, par ailleurs, dans les politiques locales de diversité en terre d'accueil, ainsi que, plus récemment, dans les politiques de coopération internationale où les organisations de migrants se voient désormais accorder un rôle considérable ${ }^{14}$. La vitalité de ces structures associatives camerounaises assure une forme de visibilité aux migrantes camerounaises en Île-de-France où bon nombre d'entre elles ont eu à diriger, dirigent et animent des associations communautaires ${ }^{15}$.

$\mathrm{Ce}$ dynamisme associatif des migrantes camerounaises comporte une originalité supplémentaire liée aux mutations sociales qui touchent leur parcours migratoire, comme le développement des unions libres, de la monoparentalité parfois stratégique et surtout des pratiques exogamiques, déjà constatées lors de nos enquêtes de terrain il y a une dizaine d'années. Leur investissement associatif s'en trouve marqué autant dans l'évolution des procédures d'adhésion, de moins en moins ethnocentriques, que dans les objectifs des actions soutenues, qui s'orientent moins vers le terroir d'origine que vers les terroirs d'alliance et/ou de résidence ${ }^{16}$. Des artistes séloignent du registre communautaire comme Princesse Erika ou les Nubians. Des actions de promotion culturelle mettent en lumière le dynamisme de femmes migrantes de culture telles que Calixthe Beyala, Léonora Miano, Julienne Zanga, Edwige Jiotsa, dont les parcours littéraires mobilisent, dans différents registres, les ressources, les espaces, les parcours et les trajectoires de migration.

\section{Le pouvoir socio-économique des associations camerounaises}

L'investissement associatif des migrantes camerounaises porte aussi sur le secteur entrepreneurial, assurant la dimension socio-économique de leur visibilité. Dans notre typologie des associations de migrants camerounais en Île-de-France ${ }^{17}$, sur cinq catégories, celles à vocation économique occupent la troisième place, après les associations dites à caractère affinitaire et les associations à caractère culturel. Elles passent ainsi devant les associations à caractère politique et celles à caractère humanitaire. C'est dire que l'action des structures associatives dans la création d'entreprise et dans la mise à disposition d'emplois est loin d'être négligeable au sein de la communauté cameL'investissement associatif rounaise en migration. La visibilité la plus immédiate qu'elle génère se perçoit à travers la création et la gestion des migrantes camerounaises porte aussi sur le secteur entrepreneurial, assurant la dimension socio-économique de leur visibilité des commerces et des restaurants exotiques ${ }^{18}$, dont un bon nombre répondent aux caractéristiques de l'économie ethnique ${ }^{19}$. Si les associations sont des cadres de mobilisation culturelle à caractère communautaire, elles sont aussi des espaces d'échange et de partage d'expériences migratoires, ainsi que des lieux d'acquisition de clientèle et de financement par les tontines pour d'éventuelles candidates à l'entreprenariat ${ }^{20}$. Elles sont également des lieux de recrutement de main-d'œuvre spécialisée (particulièrement dans le 


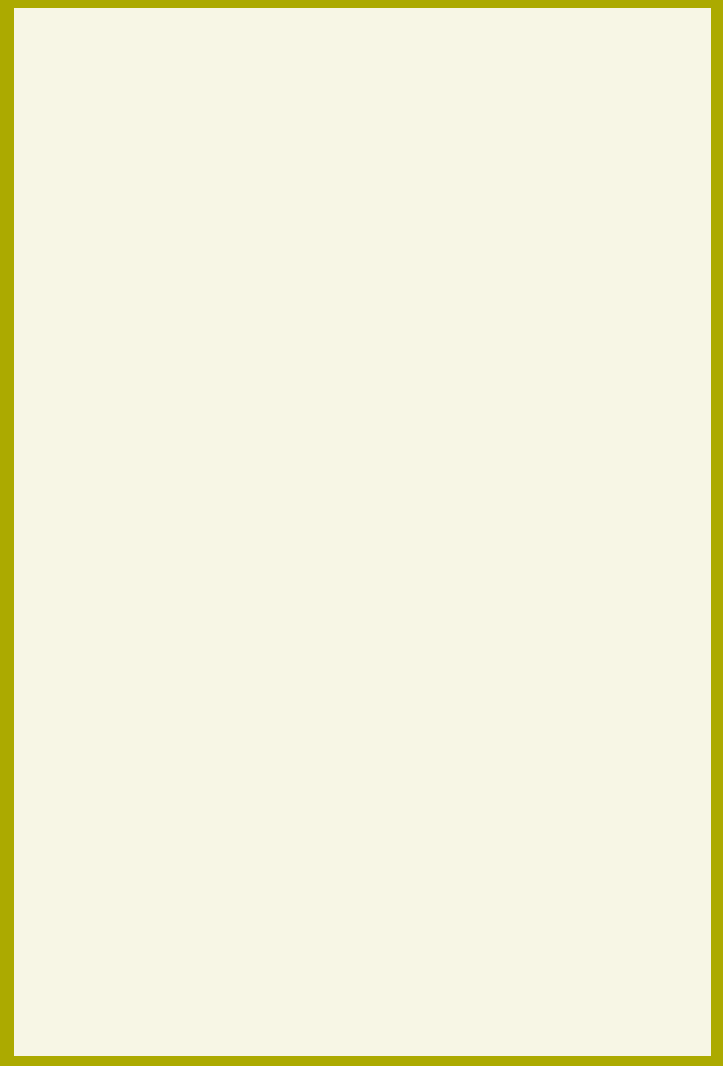

Francine: "Le pagne que m'a offert ma grand-mère la dernière fois que je l'ai vue. Je le mets en écharpe l'hiver." (c) SoPHIE PASOUET - Hans LucAs.

cadre de l'économie ethnique) et contrôlable grâce au réseau communautaire d'adhésion par le principe de don et de contre-don. Elles sont, enfin, des lieux d'échange de services divers, soutien scolaire, services traiteurs spécifiques, services à la personne, renforçant alors la cohésion de groupe au travers de réunions régulières.

Au-delà de la territorialisation en pays d'accueil, comme la rue Guy-Môquet dans le XVIIe arrondissement de Paris ${ }^{21}$, l'imbrication entre l'économique, le social et le culturel affecte les projets à destination du pays d'origine. Foires diverses, concerts et bals dansants généralement parrainés par les différents chefs traditionnels, et autres cotisations directes, sont autant de mécanismes mis en œuvre pour financer ces projets. Ces mécanismes jouent plusieurs rôles, particulièrement en ce qui concerne l'implication des migrantes dans le processus de développement de leur pays d'origine.

\section{La participation au développement du pays d'origine}

Désormais unanimement reconnues comme un canal de soutien possible au développement, les remises de fonds des migrants au titre de leur solidarité internationale ont connu une évolution considérable à travers le monde durant les quatre dernières décennies avec une perspective de croissance continue, contrairement aux aides publiques au développement. Bien que ces remises de fonds semblent ne pas assez profiter au Cameroun sur le plan macroéconomique autant quà certains pays d'émigration, elles connaissent néanmoins un accroissement tout aussi soutenu, passant d'un peu moins de 23 millions de dollars US en 1990 à plus de 251 millions de dollars US en $2014^{22}$, représentant $0,8 \%$ du PIB en provenance principalement de France et des États-Unis. La montée en puissance des migrations féminines camerounaises dès 1990, leur implication dans le développement de leur pays d'origine ainsi que leur investissement dans la structuration de l'espace associatif en migration sont certainement à prendre en considération dans l'accélération de ces transferts financiers. Cette accélération est, par ailleurs, soutenue par de nombreuses stratégies migratoires. Le financement du regroupement familial, par exemple, concerne davantage les femmes.

On peut noter également de nouvelles stratégies de départ telles que la "cybermigration maritale" 
générant des "migra-devises"23 qui participent aussi de manière non négligeable aux mécanismes de développement en pays d'origine. Ce qui rend ces migrantes de plus en plus visibles, tant dans les pays d'accueil que dans le pays d'origine où ces transferts financiers s'accompagnent aussi de transferts matériels et immatériels ${ }^{24}$ auxquels elles contribuent très activement.

Sur le plan matériel, les productions immobilières des migrants connaissent une participation féminine très remarquée, à l'instar du "quartier des Suissesses" sur la route de Mfou à Yaoundé, caractérisé par une concentration de productions immobilières effectuées par des migrantes camerounaises dont les pionnières vivent en Suisse. Dans le même temps, des intellectuelles migrantes contribuent à divers programmes de transfert de connaissances et de savoirs. Elles participent à la conception et à la réalisation de projets tels que la mise en place de l'université des Montagnes dans la région de l'Ouest-Cameroun, où l'on enregistre un nombre non négligeable d'intervenantes dans le personnel enseignant migrant en coopération et/ou en collaboration.

\section{La dimension sociopolitique de la visibilité des migrantes camerounaises}

Cette implication intellectuelle concourt à la réalisation de transferts immatériels, dont une certaine frange s'opère sur le plan idéologique, par inscription dans les mouvements d'opposition au système politique en cours dans leur pays d'origine, ou par le soutien du pouvoir en place au Cameroun. L'inscription dans l'opposition politique passe rarement par une affiliation aux divers mouvements contestataires présents dans les pays d'accueil. Elle se déploie davantage à travers les réseaux sociaux sur Internet, dans des forums spécifiques ou dans les colonnes de certains médias numériques ${ }^{25}$ par le biais d'articles satiriques signés de plumes féminines. Quant au soutien accordé au pouvoir en place à Yaoundé, il s'exprime au sein des organes extérieurs du parti gouvernant, où quelques femmes occupent bien souvent des positions importantes dans les différents bureaux de section, de sous-section et de cellule de base. Elles y impriment généralement leur marque comme dans la section FranceNord du Rassemblement démocratique du peuple camerounais (RDPC), parti au pouvoir. Elles s'y distinguent par la mise en place d'une structure associative dénommée "Femmes dynamiques", dont l'objectif est non seulement de mieux lier leur action politique aux idéaux sociaux spécifiques de genre, mais aussi d'harmoniser leurs démarches afin de mieux défendre leurs intérêts au sein d'un parti qui demeure encore sous une écrasante domination masculine.

\section{Conclusion}

Les conditions de mise en migration originellement peu favorables aux femmes dans le courant migratoire camerounais n'ont fait que retarder leur arrivée dans les espaces migratoires. Les migrantes camerounaises ancrent prioritairement leur visibilité dans des postures collectives en mobilisant des acquis prémigratoires, plus particulièrement d'ordre culinaire, pour structurer des pratiques de groupe. Il s'agit d'une démarche citoyenne féminine mobilisée dans un processus de construction diasporique, dont elles assurent la patrimonialisation à diverses échelles, y compris dans les engagements politiques généralement orientés vers le pays d'origine. 